\title{
A Detailed Process for Aviation Systems Requirements Analysis and Definition via Model-Based Approach
}

\author{
Teng Li
}

\begin{abstract}
Due to the system complexity, it is difficult to define system requirements clearly and correctly by natural languages. Although engineers spend plenty of time on system requirements analysis, system requirements still suffer some drawbacks. In the paper, a new and powerful approach is proposed to analyze and define system requirements. The model-based approach was utilized to analyze system characteristics and then define system requirements of a helicopter air conditioner system. In this process, System Modeling Language was used to build the model to analyze and describe the system characteristics through different views. And qualified system requirements of the helicopter air conditioner system, could be derived effectively by the model.
\end{abstract}

Keywords System requirements · Model-based approach · System modeling language $\cdot$ Helicopter air conditioner system

T. $\operatorname{Li}(\otimes)$

Avic-Digital CO., Ltd., Jingshunlu No. 7, Chaoyang District, Beijing, China

(C) The Author(s), under exclusive license to Springer Nature Switzerland AG 2021 